\title{
Primary Management of Large Pancreatic Pseudoaneurysm by Ultrasound-Guided Percutaneous Thrombin Injection after Failure of Transarterial Pseudoaneurysm Embolization
}

\author{
Pawan Kumar Garg ${ }^{1}$ Pushpinder Singh Khera ${ }^{1}$ Rengarajan Rajagopal ${ }^{1}$ Neeraj Mehta ${ }^{1}$ \\ Swarnava Tarafdar ${ }^{1}$ \\ ${ }^{1}$ Department of Radiology, All India Institute of Medical Sciences, \\ Jodhpur, Rajasthan, India \\ J Clin Interv Radiol ISVIR 2017;1:130-133. \\ Address for correspondence Pawan Kumar Garg, MD, DNB, \\ Department of Radiology, All India Institute of Medical Sciences, Basni \\ Industrial Area Phase II, Jodhpur 342005, Rajasthan, India \\ (e-mail: drgargpawan@gmail.com).
}

\begin{abstract}
Keywords

- pancreatic pseudoaneurysm

- percutaneous thrombin injection

- pseudoaneurysm embolization

Pancreatic pseudoaneurysm is a rare but life-threatening complication secondary to pancreatitis, surgery, trauma, or infection. Early treatment is advocated due to high risk of rupture and bleeding. Surgical treatment has traditionally been the first line but is associated with high morbidity. Transarterial embolization or exclusion is the treatment of choice; however, in cases with failure of endovascular treatment, percutaneous thrombin injection can be used successfully. We present a case of a large pancreatic pseudoaneurysm managed successfully with percutaneous ultrasound-guided thrombin injection after failure of transarterial management.
\end{abstract}

\section{Case Report}

A 24-year-old male nonalcoholic patient with acute pancreatitis presented to the hospital with severe abdominal pain, nausea, and vomiting. At the time of presentation, his serum amylase and lipase levels were elevated with normal liver and renal function. Duplex Doppler (-Fig. 1a) showed the presence of a large pseudoaneurysm with to and fro color flow and characteristic yin-yang pattern in the pancreatic head region. Multiphase contrastenhanced computed tomography (CT) of the abdomen ( $\mathbf{- F i g}$. 1b-d) demonstrated a pseudoaneurysm in the pancreatic head region, measuring $63 \times 46 \times 40 \mathrm{~mm}$ with a thin rim of peripheral thrombus. In addition, features of pancreatitis were noted as well as a large pseudocyst in the pancreatic tail causing displacement of the stomach anteriorly.

As open surgery was not the best option in acute pancreatitis, transarterial embolization was considered. Digital subtraction angiography ( $\mathbf{- F i g . ~ 2 )}$ ) of the common hepatic artery showed the presence of a large pseudoaneur$\mathrm{ysm}$ in the pancreatic head region, at the origin of the gastroduodenal artery. Despite superselective angiography of the gastroduodenal artery and proper hepatic artery in different projection, the neck of the pseudoaneurysm could not be identified. Since we were not able to identify the neck and the feeding artery to the pseudoaneurysm, transarterial embolization was not considered and percutaneous embolization with thrombin injection was chosen. A spinal needle $(22 \mathrm{G}$ ) was inserted into the aneurysmal sac through the anterior abdominal wall under ultrasound (US) guidance (- Fig. 3a) and 1,000 IU of reconstituted human thrombin (ReliStat, Reliance Life Science) was injected. US demonstrated thrombus formation within the aneurysmal sac and at the end of injection, there was complete obliteration of the sac with no color flow seen. A repeat angiogram ( $\mathbf{- F i g . 3 b}$ ) through the common hepatic artery showed no filling of the aneurysm. No immediate complications were observed. Follow-up abdominal CT ( - Fig. 4b, c) at 1 week and Doppler US ( - Fig. 4a) examination at 4 weeks confirmed complete thrombosis of the pseudoaneurysm with preserved flow through celiac arterial branches. 


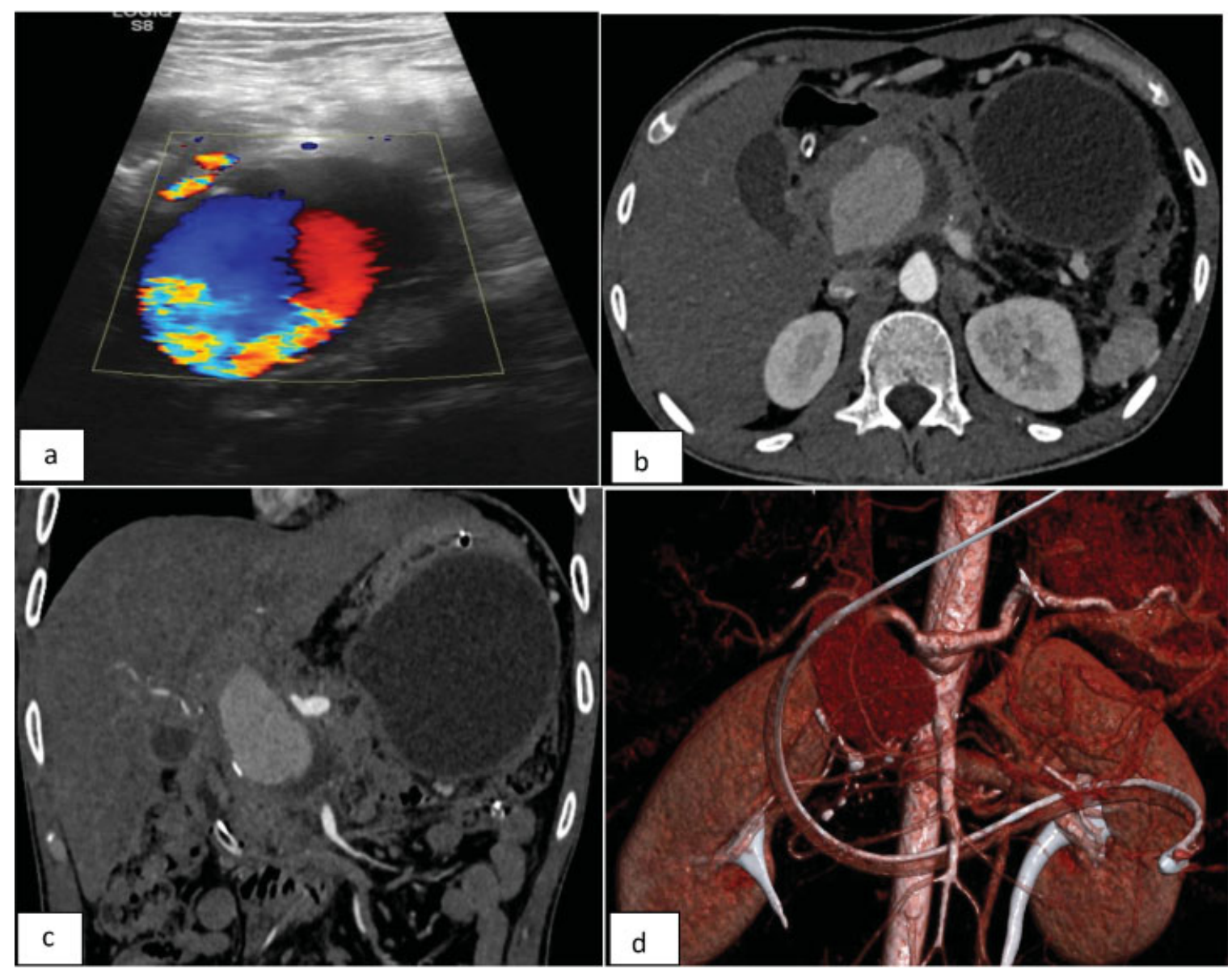

Fig. 1 (a) Ultrasound with color Doppler showing large pseudoaneurysm with characteristic yin-yang color flow. CT angiography of abdomen in (b) axial and (c) coronal planes and (d) VRT images show large arterial phase enhancing pseudoaneurysm in the pancreatic head region. Also, presence of a large pseudopancreatic cyst in the pancreatic tail region causing displacement of the stomach anteriorly. CT, computed tomography; VRT, volume rendering technique.

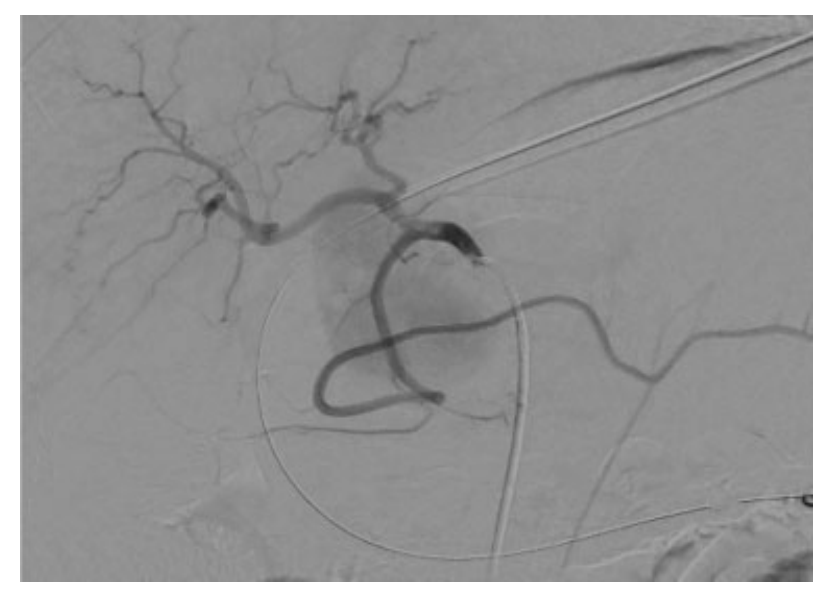

Fig. 2 DSA image with catheter tip at the origin of the common hepatic artery shows filling of a large pseudoaneurysm with nonvisualization of a neck. DSA, digital subtraction angiography.

\section{Discussion}

Pseudoaneurysms have been reported in almost all the visceral arteries but are most commonly seen in the splenic artery (46\%), followed by the renal (22\%), hepatic (16.2\%), pancreaticoduodenal (1.3\%), and gastroduodenal artery (1.5\%). ${ }^{1}$ Pancreatic pseudoaneurysm is a rare complication of pancreatitis with lethal outcome if ruptured. Other causes include surgery, trauma, and infection. In pancreatitis, the most commonly affected artery is the splenic artery followed by the gastroduodenal artery and the pancreaticoduodenal arcade. ${ }^{2}$ Mortality secondary to the pseudoaneurysm depends on duration of the pancreatitis and the vessels involved. If ruptured, mortality rates range from 15 to $50 \%{ }^{3}$

CT angiography of the abdomen is the investigation of choice to assess the severity of pancreatitis, arterial source of the pseudoaneurysm, and associated complications such as the pseudopancreatic cyst. Treatment of pancreatic pseudoaneurysm is either surgical or endovascular. Surgery is traditionally the first-line treatment but is invasive, more challenging, associated with high morbidity and therefore indicated in hemodynamically unstable patients, failed/recurrent pseudoaneurysm after transarterial treatment, and in the presence of necrotizing pancreatitis.

Transarterial endovascular treatment includes embolization using coils, N-butyl cyano acrylate, gelfoam, stent graft, and vascular plug. Transarterial coil placement is preferred with a reported success rate of 92 to $97 \%$ and lower overall 


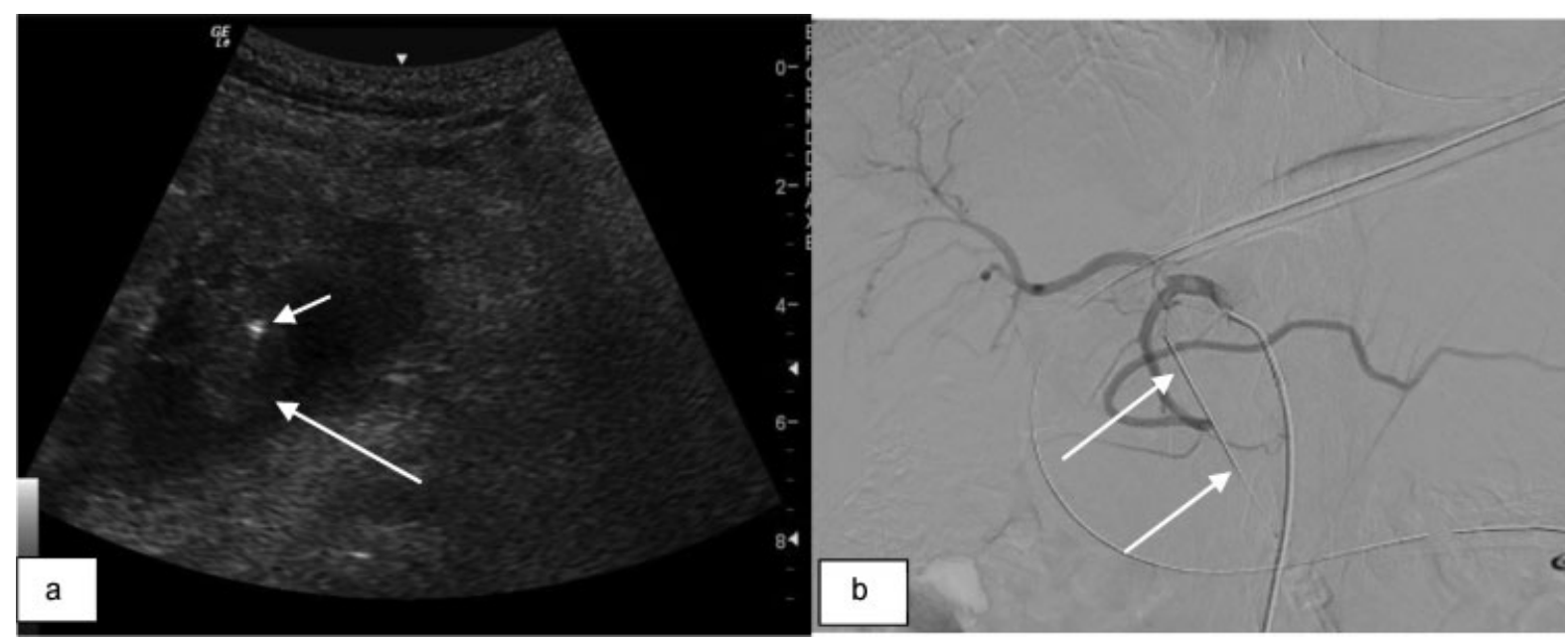

Fig. 3 (a) Ultrasound image at the time of percutaneous thrombin injection shows needle tip (small arrow) in the aneurysmal sac with surrounding hematoma (large arrow). (b) DSA image after completion of thrombin injection while the needle (white arrow) remained within the sac shows complete obliteration of the aneurysm sac. DSA, digital subtraction angiography.

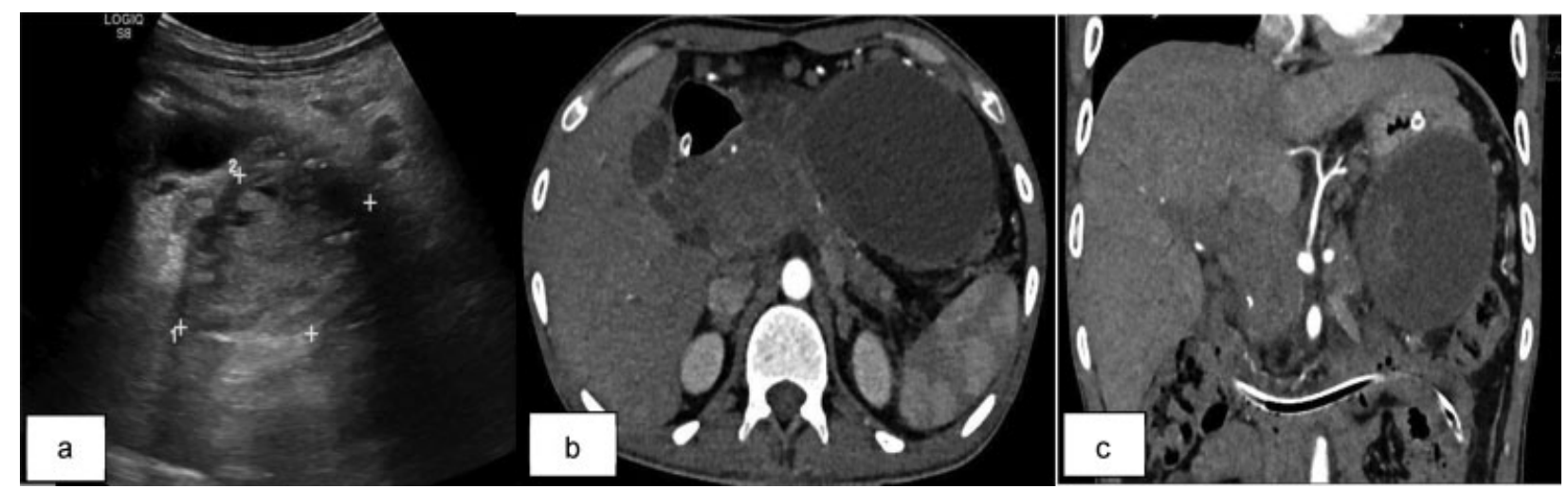

Fig. 4 (a) Follow-up ultrasound and CT images in (b) axial and (c) coronal planes show complete obliteration of the pseudoaneurysm. CT, computed tomography.

rate of serious complications. ${ }^{4,5}$ Coil embolization of the aneurysm can be performed if entry into the sac is possible and the neck of aneurysm is narrow. Coiling of the feeding artery is another option especially if the pseudoaneurysm is arising from the gastroduodenal artery. N-butyl cyanoacrylate and gelfoam can be used either alone or in association with coiling.

In our case, the neck of the pseudoaneurysm could not identified despite obtaining angiography on different projections, hence aneurysmal coiling was not performed. Also, the feeding artery could not be identified. An alternative option to transarterial management of the pseudoaneurysm is the percutaneous embolization by thrombin injection under US or CT guidance.

Thrombin injection is traditionally used for the treatment of an iatrogenic femoral artery pseudoaneurysm. In 1986, Cope and Zeit first reported the use of percutaneous thrombin injection for the treatment of aneurysms. ${ }^{6}$ In recent years, it is also used for percutaneously accessible visceral artery pseudoaneurysm. ${ }^{7}$ One report described the use of thrombin injection for treatment of a large pseudoaneurysm measuring $60 \times 44 \times 45 \mathrm{~mm}$ and arising from the superior mesenteric artery. ${ }^{8}$ Thrombin is a clotting factor formed from prothrombin and allows polymerization of fibrinogen to form fibrin. Percutaneous injection of thrombin is safe if it is injected slowly according to the flow and size of the pseudoaneurysm. Nontarget embolization is one of the common complications associated with thrombin injection. The other potential complication associated with thrombin injection is anaphylaxis; however, with the use of human thrombin, the risk of anaphylaxis has markedly reduced. ${ }^{9}$ Furthermore, US-guided injection allows real-time visualization of the needle track and reduces the risk of accidental puncture of the bowel, vessels, and organs. The incidence of recurrent hemorrhage following embolization of pancreatic pseudoaneurysms has been reported to be as high as 30\%; therefore, long-term follow-up is necessary in these patients. $^{10}$

In conclusion, percutaneous US-guided thrombin injection could be the first-line treatment following failure of transarterial embolization or when cannulation or identification of the feeding arteries of the pseudoaneurysm is difficult. 


\section{Conflict of Interest}

None.

\section{References}

1 Deterling RA Jr. Aneurysm of the visceral arteries. J Cardiovasc Surg (Torino) 1971;12(04):309-322

2 Ammori BJ, Madan M, Alexander DJ. Haemorrhagic complications of pancreatitis: presentation, diagnosis and management. Ann R Coll Surg Engl 1998;80(05):316-325

3 Manazer JR, Monzon JR, Dietz PA, Moglia R, Gold M. Treatment of pancreatic pseudoaneurysm with percutaneous transabdominal thrombin injection. J Vasc Surg 2003;38(03): 600-602

4 Gabelmann A, Görich J, Merkle EM. Endovascular treatment of visceral artery aneurysms. J Endovasc Ther 2002;9(01): $38-47$
5 Tulsyan N, Kashyap VS, Greenberg RK, et al. The endovascular management of visceral artery aneurysms and pseudoaneurysms. J Vasc Surg 2007;45(02):276-283, discussion 283

6 Cope C, Zeit R. Coagulation of aneurysms by direct percutaneous thrombin injection. AJR Am J Roentgenol 1986;147(02): 383-387

7 Kang SS, Labropoulos N, Mansour MA, et al. Expanded indications for ultrasound-guided thrombin injection of pseudoaneurysms. J Vasc Surg 2000;31(02):289-298

8 Barbiero G, Battistel M, Susac A, Miotto D. Percutaneous thrombin embolization of a pancreatico-duodenal artery pseudoaneurysm after failing of the endovascular treatment. World J Radiol 2014; 6(08):629-635

9 Elford J, Burrell C, Freeman S, Roobottom C. Human thrombin injection for the percutaneous treatment of iatrogenic pseudoaneurysms. Cardiovasc Intervent Radiol 2002;25(02):115-118

10 Coll DP, Ierardi R, Kerstein MD, Yost S, Wilson A, Matsumoto T. Aneurysms of the pancreaticoduodenal arteries: a change in management. Ann Vasc Surg 1998;12(03):286-291 